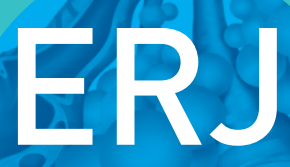

open research

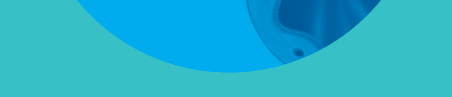

\section{A frequent phenotype for paediatric sleep apnoea: short lingual frenulum}

\author{
Christian Guilleminault, Shehlanoor Huseni and Lauren Lo
}

Affiliation: Stanford University Sleep Medicine Division, Redwood City, CA, USA.

Correspondence: Christian Guilleminault, Stanford University Sleep Medicine Division, 450 Broadway, (Pavillion C 2nd floor), Redwood City, CA 94063, USA. E-mail: cguildastanford.edu

ABSTRACT A short lingual frenulum has been associated with difficulties in sucking, swallowing and speech. The oral dysfunction induced by a short lingual frenulum can lead to oral-facial dysmorphosis, which decreases the size of upper airway support. Such progressive change increases the risk of upper airway collapsibility during sleep.

Clinical investigation of the oral cavity was conducted as a part of a clinical evaluation of children suspected of having sleep disordered breathing (SDB) based on complaints, symptoms and signs. Systematic polysomnographic evaluation followed the clinical examination. A retrospective analysis of 150 successively seen children suspected of having SDB was performed, in addition to a comparison of the findings between children with and without short lingual frenula.

Among the children, two groups of obstructive sleep apnoea syndrome (OSAS) were found: 1) absence of adenotonsils enlargement and short frenula $(n=63)$; and 2) normal frenula and enlarged adenotonsils $(\mathrm{n}=87)$. Children in the first group had significantly more abnormal oral anatomy findings, and a positive family of short frenulum and SDB was documented in at least one direct family member in 60 cases.

A short lingual frenulum left untreated at birth is associated with OSAS at later age, and a systematic screening for the syndrome should be conducted when this anatomical abnormality is recognised.

@ERSpublications

A short lingual frenulum left untreated at birth is associated with obstructive sleep apnoea syndrome at a later age http://ow.ly/6kMQ30163nG 


\section{Introduction}

Obstructive sleep apnoea syndrome (OSAS) is related to the abnormal collapse of the upper airway during sleep. This abnormal collapsibility in both children and adults has been related to sleep, which causes fundamental modifications to the pharyngeal muscle tone and reflex responses; position, given that sleep usually occurs in a recumbent position; and intrinsic and extrinsic factors: the upper airway has an intrinsic collapsibility that is studied by evaluation of the "critical pressure", and extrinsic factors may lead to increased collapsibility. Three external factors impacting the retropalatal and retroglossal space of the upper airway have been firmly established: upper airway fat deposits; non-fat-related hypertrophy of upper airway tissues in which chronic inflammation is a factor; and craniofacial features impacting the upper airway size, possibly related to genetic and environmental factors. Genetic mutations present at birth may not impact the upper airway until later in life. Environmental factors have a variable effect: the speed of their impact on upper airway collapsibility may be slow, and these factors may be present for a long time before the onset of clinically observed sleep disordered breathing (SDB), which may result in clinical symptoms only recognised during adulthood despite the presence of anatomical and/or functional anomalies during childhood. In addition, environmental factors interact with the genetic constitution of the individual, impacting the expression of a genetic trait [1].

We have already reported several factors that impact normal growth of the oral-facial structures leading to the development of OSAS in both children and adults [2-4]. Our study addresses one of these risk factors, which has not yet been linked to the development of OSAS. It investigates the association between a short lingual frenulum and OSAS.

Normally at birth, the tongue is placed high in the palate, and its continuous activity related to sucking, swallowing and masticating induces stimulation of the intermaxillary synchondrosis [1], which is active until 13-15 years of age, leading to normal oral-facial growth. Normal nasal breathing is associated with this tongue position.

A short lingual frenulum has been associated with sucking and swallowing difficulties early in life, leading to "clipping" of the frenulum in the newborn [5-8]. In older children speech difficulties have been related to an untreated short frenulum [8-10]; it was also shown to lead to mouth breathing with modification of the position of the tongue and secondary orthodontic impacts resulting in an anterior and posterior crossbite, a disproportionate growth of the mandible and an abnormal growth of the maxilla $[8,10,11]$. All these anatomical changes impact the size of the upper airway and increase the risk of its collapse during sleep.

Recognition of the negative role of a short lingual frenulum led myofunctional specialists to develop sophisticated protocols to investigate infants and children with short lingual frenulua [12, 13], but the association between a short lingual frenulum and OSAS is currently unrecognised.

"Clipping" of the short lingual frenulum is still proposed when difficulties are recognised during very early infancy, but if a simple clipping is performed after the first few months of life, the long-term results are reported as unpredictable [14], with persistence of an abnormal short lingual frenulum.

This retrospective study on anonymised data was approved by the Stanford University institutional review board. It investigates the association between a short lingual frenulum and OSAS in children.

\section{Method}

The investigation is a retrospective study of children aged 3-12 years who were referred to our clinic between January 1, 2014 and August 1, 2015 for "sleep disorders". Syndromic children, children with neuropsychiatric syndromes, chronic medical conditions and obesity were not included in this survey. The presented children may have initially been referred for diagnosis of OSAS, for other sleep-related complaints, or for a post-treatment follow-up after a diagnosis of OSAS (adenotonsillectomy in most cases).

\section{Definition and measurement of a short lingual frenulum}

The lingual frenulum is a vestigial embryological element that is mostly fibrous in its consistency, a result of adhesion between the tongue and the floor of the mouth during embryogenesis. Apoptosis controlled by genes separates the tongue from the primitive pharynx during embryogenesis $[12,15]$.

Recommendations in the literature are to measure the interincisive distance with maximum mouth opening and the tongue low-placed and with the tip of the tongue placed against the palate, measuring the percentage difference between the two measurements, and to measure the distance from the insertion of the frenulum at the base of the tongue to the tip of the tongue. KotLow $[16,17]$ measures the "free tongue", which is defined as "the length of tongue from the insertion of the lingual frenum into the base of the tongue to the tip of the tongue", and considers a normal frenulum length to be $>16 \mathrm{~mm}$. RuFFoli et al. [18] measure the length of the frenulum itself and classify a normal frenulum length in children aged $\geqslant 6$ years to be $>20 \mathrm{~mm}$ with a mild problem at $16-19 \mathrm{~mm}$. These were our references, with $>16 \mathrm{~mm}$ our cut-off point for a normal free-tongue length for children aged $\geqslant 3$ years $[16,17]$. 


\section{Data collection}

All children sent to the clinic underwent a systematic clinical evaluation following pre-established guidelines. The evaluation consisted of a paediatric general evaluation, a clinical sleep medicine evaluation including the paediatric sleep questionnaire and the paediatric daytime sleepiness scale $[19,20]$, completed by the parents or the child, based on age. Evaluation of possible daytime consequences included diminished alertness, daytime sleepiness and fatigue, cognitive and memory problems, behavioural changes including those evoking cataplexy or seizure disorders, and social impairment. Past medical history was systematically investigated with questions on problems in early infancy regarding sucking, swallowing or masticating, difficulty with elocution and any speech treatment later, history of nasal allergies, abnormal mouth breathing, adenotonsillectomy, teeth problems including teeth agenesis, abnormal growth or early extraction and orthodontic treatment. Family history of short frenula, OSA and symptoms evoking a short frenulum were systematically obtained, and the oral-facial presentation of the family member bringing the child to clinic was systematically evaluated. If a short lingual frenulum in a direct family member was reported, efforts were made to have the family member checked at a follow-up visit of the child.

Standardised scales (the Friedman tonsil scale, the Mallampati-Friedman scale and the "facial harmony" scale) [21-24] and subjective scales, as previously outlined [15] were used during the clinical evaluation.

To evaluate the frenulum, our systematic examination used manoeuvres defined and reported by myofunctional therapists, evaluating for placement of tongue at rest; capability of using the tongue to perform manoeuvres such as trying to touch the nose and chin with the tip of the tongue; making a "cigar tongue" while protruding the tongue; touching the median raphe with the tip of the tongue while maintaining a wide, open mouth and observing whether there is a closing of the upper jaw to perform the manoeuvre; and ability to pronounce some letters and vowels. The opening of the mouth is measured using calipers, with measurement of the maximum opening from the lower left incisor to the upper left incisor, and then measurement with mouth open wide and the tongue tip touching the incisive papilla. This measurement was considered normal if the difference between the two measurements was $<50 \%$.

The position of the frenulum was examined and it was considered abnormal if it was attached underneath the tongue at any point between the tongue mid-point and the apex or inserted under the tongue as in a normal subject but short, not reaching the apex. The shape of the tip of the tongue was also noted, indicating a heart shape, a V shape or square. Finally, palpation of the region below the median region of the buccal floor with the bilateral index fingers was used to reveal abnormal genioglossal muscle resistance.

Measurements of the length of the frenulum were performed by measuring between two points; measurements were performed twice: when the short frenulum was recognised and at the end of the clinical evaluation. The mean of the two measurements was recorded in millimetres. All measurements were performed by the same individual.

For the study, we only took into consideration the measurement of the length of the free tongue (i.e. the mean of the two measurements performed at time of evaluation), despite the fact that we also measured the length of the frenulum as described by RufFoli et al. [18] (fig. 1).

All children who were suspected of having abnormal breathing during sleep based on the total clinical evaluation, independent of a short lingual frenulum, were recommended to undergo polysomnography (PSG) in a sleep laboratory.

The following variables were systematically monitored during nocturnal sleep: four electroencephalogram leads, two electro-oculogram leads, chin and leg electromyogram (EMG) leads and one ECG lead; respiration was monitored using a nasal cannula pressure transducer, a mouth thermistor, thoracic and abdominal inductive plethysmography bands, a finger oxygen saturation oximeter (Masimo, Irvine, CA, USA) with derivation of oximetry and finger plethysmography signals, a neck microphone, diaphragmatic-intercostal abdominal muscle EMGs and a transcutaneous carbon dioxide electrode; leg EMGs were also monitored. Children were continuously video-monitored during the recording and had one parent present.

\section{Analyses}

The different clinical scales were tabulated. The different results of the frenulum evaluation were considered as "indicative", but the only variable interred in the analysis was the mean measured length of the free tongue distance from the frenulum. Sleep and respiratory scoring followed the recommendations of the American Academy of Sleep Medicine (AASM) with hypopnoea scored for either a 3\% drop in oxygen saturation or an arousal response [26]. Nasal inspiratory flow limitation [27] was determined using published criteria $[28,29]$. The time spent mouth breathing was also calculated as a percentage of total sleep time [30]. The different scoring analyses followed AASM recommendations [26]. 

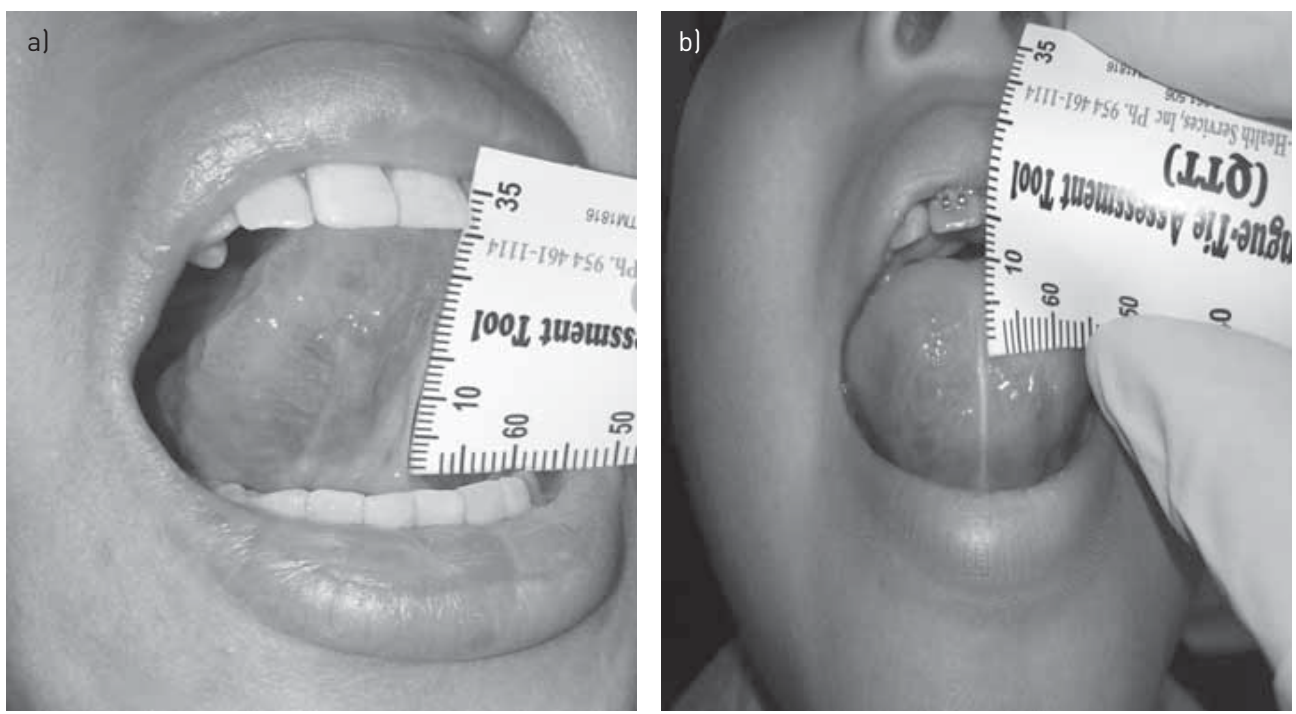

FIGURE 1 Measurement of the frenulum using the commercially available Quick Tongue Tie Assessment Kit (Neo Health Services Inc., Coconut Creek, FL, USA). a) Normal frenulum [9]; b) free tongue [7]. Complete clinical protocols for lingual frenulum investigations for infants [13] and children-adolescents [25] have been published.

All variables were anonymised and submitted for statistical analysis. The continuous variables are presented as mean $\pm \mathrm{SD}$. A normality test was performed on all continuous variables. If the variables were distributed normally, the paired t-test was used; otherwise, the Wilcoxon rank sum test was computed. A p-value $<0.05$ was considered statistically significant.

\section{Results}

As mentioned, children with neuromuscular disorders, major psychiatric disorders, craniofacial syndromes, major paediatric illnesses (cystic fibrosis, cancers and haematological syndromes such as sickle cell anaemia) and obesity based on body mass index $\left(>28 \mathrm{~kg} \cdot \mathrm{m}^{-2}\right)$ were excluded from the review.

The data from 150 successively seen children diagnosed with OSAS and with complete charts including PSG results are presented. The children were subdivided into the short lingual frenulum group or the normal lingual frenulum group (based on the short lingual frenulum definition described earlier). In children aged $\geqslant 6$ years, we found no difference in classification using KotLow $[16,17]$ or RufFoli et al. [18] methods: children considered to have a short frenulum were classified as such using both measurements. There was no attempt to define other abnormal aspects of the frenulum (e.g. thickness or localisation of the insertion of the frenulum on the tongue) [15] (fig. 2).

Table 1 presents the demographic, clinical, anatomical and PSG findings for all the subjects and for the subgroups with short and normal lingual frenulum. 63 children were in the short frenulum group (aged $9.88 \pm 3.21$ years) and 87 children were in the normal frenulum group (aged $8.05 \pm 3.59$ years). Some degree of snoring was reported by parents in $39(62 \%)$ of the children with abnormal frenula and in $59(66 \%)$ children with normal frenula (nonsignificant). Children did not differ in levels of complaints of daytime sleepiness, fatigue or inattention-hyperactivity (the most common complaints from the subjects).

The two groups differed significantly in the anatomical description of the oral cavity (table 1), with the short frenulum group having significantly more frequent reports of a "high and narrow palatal vault" and scores of 4 on the Mallampati-Friedman scale $(0-4)(p=0.0001)$, while the normal frenulum group had a significantly greater frequency of scoring $\geqslant 4$ on the Friedman tonsil scale $(p=0.0001)$. The mean tonsil size score was 1.8 in the abnormal frenulum group versus 3.2 in the other children. Five children in the short lingual frenulum group had had adenotonsillectomies that had not resolved the OSA, but the short lingual frenulum had been missed at time of the surgery.

A Wilcoxon signed-rank test revealed statistical significance between the average apnoea/hypopnoea index $(\mathrm{AHI})$ of $13.06 \pm 4.17$ in the short frenulum group versus $11.36 \pm 5.39$ in the normal frenulum group $(p=0.025)$. The short lingual frenulum was predictive of a higher AHI in males $(p=0.0069)$, but not in females $(\mathrm{p}=0.8615)$. The average oxygen saturation in the short frenulum group was $89.42 \pm 1.52 \%$ versus $89.85 \pm 1.52 \%$ (not significant). Again, a short frenulum was only predictive of lower arterial oxygen saturation measured by pulse oximetry $\left(\mathrm{SpO}_{2}\right)$ nadir in males $(\mathrm{p}=0.0060)$, but not in females $(\mathrm{p}=0.6089)$. 

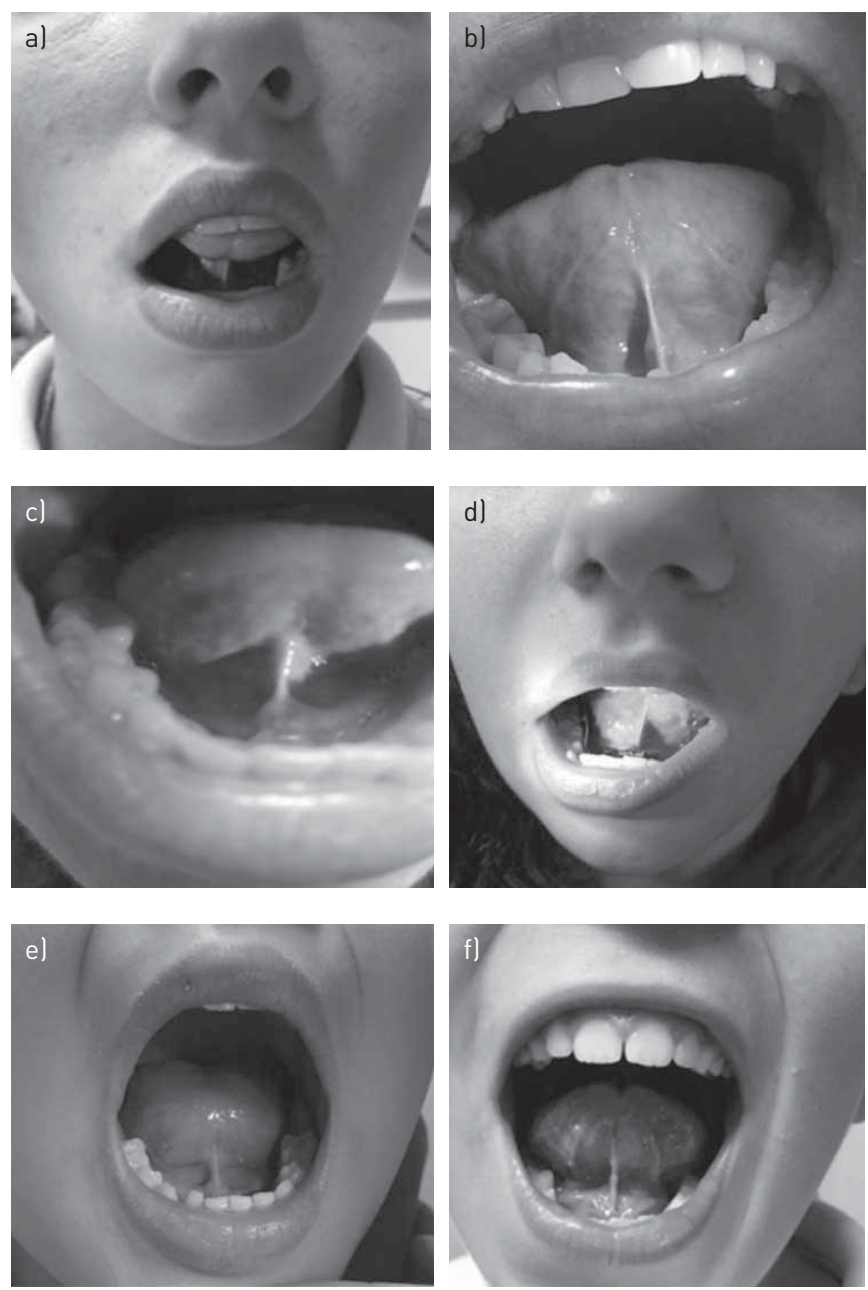

FIGURE 2 a-h) Examples of short frenula in children and teenagers. Consideration was given only to shortness of the frenulum and not difference in presentation of the frenulum. Histological studies have shown that different types of fibres may be present, depending on the individual. Short frenula were found in e) a 40-year-old mother and f) her 14-year-old daughter. The other subjects are aged 3-16 years. All subjects have obstructive sleep apnoea syndrome.
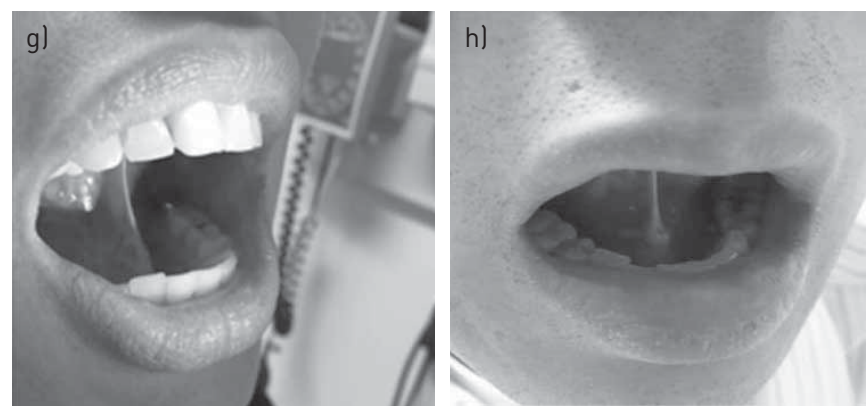

A two-tailed t-test revealed that patients with short lingual frenulae were found to be significantly older $(\mathrm{p}=0.0015)$. In addition, qualitative analysis of the age distribution of the short lingual frenulum group shows a unimodal normal distribution of age around a mean of $9.8 \pm 3.2$ years, whereas the age distribution the normal frenulum group was more evenly distributed across the spectrum ( $8.05 \pm 3.6$ years).

A univariate analysis revealed that age, sex and short lingual frenulum were all found to be statistically significant predictors of OSAS severity based on AHI and $\mathrm{SpO}_{2}$ nadir. The association between frenulum abnormality and OSAS severity was more notable among males than females. However, a multivariate analysis with least squares regression analysis showed that age was the only independent predictor of OSAS severity in this series.

The frenulae in all the adults who accompanied the child during the consult were systematically measured; if the family history revealed the presence of SDB in a member not present at the initial evaluation, a request to have the individual present at a follow-up visit was made. This systematic request revealed the presence of a short lingual frenulum in a parent or sibling of 60 out of the 63 index cases in the short 


\begin{tabular}{|c|c|c|c|c|}
\hline & Subjects & Short frenulum & Normal frenulum & p-value \\
\hline Subjects & 150 & 63 & 87 & \\
\hline Age years mean \pm sD $(n / N)$ & 150 & $9.88 \pm 3.21(63 / 150)$ & $8.05 \pm 3.59(87 / 150)$ & 0.0015 \\
\hline Females & 58 & $29 / 63(46)$ & 29/87 (33) & 0.1288 \\
\hline \multicolumn{5}{|l|}{ Symptoms } \\
\hline Fatigue & 147 & $61 / 63(96)$ & $86 / 87$ (98) & 0.5725 \\
\hline EDS & 73 & $35 / 63(55)$ & $38 / 87(43)$ & 0.1859 \\
\hline Inattention/hyperactivity & 90 & $43 / 63(68)$ & 47/87 (54) & 0.0926 \\
\hline \multicolumn{5}{|l|}{ Anatomy } \\
\hline High and narrow palatal vault & 63 & $56 / 70(80)$ & $7 / 80(8.75)$ & 0.0001 \\
\hline Friedman tonsil score & 150 & $1.8 \pm 0.9$ & $3.2 \pm 0.9$ & 0.0001 \\
\hline Mallampati scale score & 150 & $3.4 \pm 0.6$ & $2.9 \pm 0.7$ & 0.0001 \\
\hline Past medical history & 150 & & & \\
\hline Difficulty sucking & & 6 & 0 & \\
\hline Difficulty swallowing & & 4 & 0 & \\
\hline Speech problems & & 31 & 0 & \\
\hline
\end{tabular}

Data are presented as $n, n / N(\%)$ or mean $\pm S D$, unless otherwise stated. Feeding and swallowing difficulties were poorly recollected, except in a few cases where the problem was mentioned as "important"; the speech problems were better recalled and were described as "lisp", "stutter" or having led to speech therapy, mostly in school $(n=15)$. Despite speech therapy, the presence of a short lingual frenulum had not been investigated or mentioned to parents. EDS: excessive daytime sleepiness.

frenulum group. The other family members identified as having a short lingual frenulum presented with symptoms or were already being treated for SDB. The short frenulum group included Caucasians $(n=34)$, Far-East Asians ( $n=23$ ) (the two prominent ethnic groups in the clinic), but also five Mexican-Indians and one African-American (the least represented group in the clinic), indicating the presence of multiethnicity in the short lingual frenulum group.

\section{Discussion}

Experiments using monkeys as subjects have demonstrated a continuous interaction between mouth breathing, functions of the tongue and oral-facial growth $[31,32]$. The interaction between abnormal bone growth stimulation and the absence of nasal breathing with secondary development of mouth breathing is responsible for an abnormal development of the oral-facial bone structures supporting the upper airway, thus increasing the risk of upper airway collapse during sleep [30]. The abnormal oral-facial growth leading to a reduction of the ideal size of the upper airway will occur at a variable speed, depending on the individual, and abnormal breathing during sleep occurs over time, initially with flow limitation then with progressive worsening toward full-blown OSAS. A short lingual frenulum has been shown to lead to mouth breathing and to abnormal development of the oral cavity $[8,11,12]$, increasing the risk of upper airway collapsibility during sleep; interestingly, children with short frenulae in our study were recognised as having SDB later than children who had enlarged tonsils.

Systematic investigation of a short frenulum at birth and clipping in early infancy varies greatly depending on the geographic location of delivery. A short frenulum may lead to speech problems of variable types [8-10, 33], but as shown in our group, children may have had speech therapy without the investigation of the presence of a short frenulum, or an inappropriate treatment of the short lingual frenulum may have been performed. Usually when a child is recognised with speech problems, mouth breathing is commonly associated with either day or night or only during sleep, and speech impairment and mouth breathing (particularly mouth breathing during sleep), will need simultaneous treatment $[4,14]$.

Our study found a large number of children with both OSAS and a short lingual frenulum. As several siblings with short frenulum and suspicion of OSAS were monitored during the same period, this may have increased the numbers of positive findings, but we only had 12/63 siblings in our survey leaving at least 51 families with short lingual frenulum and OSAS. However, our study is not a general population study and is performed on a specific at-risk group, which is a limitation.

In $95 \%$ of our index cases (no family investigation existed for the last 5\%), short frenulae were documented upon examination of a direct family member with different clinical presentations. Why short lingual frenula run in families is unknown [34]. Ankyloglossia is considered a congenital anomaly reported 
in $4-5 \%$ of the general population [34]. It may be inherited as an autosomal X-linked dominant trait (more common in males). A short lingual frenulum has been reported in genetically related syndromes such as Beckwith-Wiedemann, orofacial digital syndrome, cleft palate and Optiz syndrome, but all mutations associated with these syndromes are not known. Genetic studies have been performed on subjects having ankyloglossia and cleft palate relating to a mutation on the TBX22 gene (a T-box transcription factor) and on mice with ankyloglossia looking at the involvement of LGR5, an orphan G-protein coupled receptor [35-38]. Females with such mutations may present with short lingual frenula alone. It is possible that the short frenulum is associated with a malposition of the tongue due to an unknown mutation, but we have no proof to support such a hypothesis.

In our group of children aged $\geqslant 3$ years, we found the presence of a short lingual frenulum that had been left untreated despite indications of clinical problems earlier in life in 41 out of 63 subjects. As reported in prior studies, we observed both mouth breathing and abnormal anatomical findings at the time of recording and diagnosis of OSAS. When considering results of several of our investigations performed in children a pattern emerges: a dysfunction early in life involving abnormal nasal breathing, sucking and masticating leads to progressive dysmorphoses favouring increased collapsibility of the upper airway during sleep, which worsens with ageing and leads to the development of SDB over time up to adulthood.

\section{Acknowledgements}

We thank Soroush Zaghi (Stanford University Sleep Medicine Division, Redwood City, CA, USA) for his help with the statistical analyses.

\section{References}

1 Guilleminault C, Akhtar F. Pediatric sleep-disordered-breathing: new evidence on its development. Sleep Med Rev 2015; 24: 46-56.

2 Huang YS, Guilleminault C. Pediatric obstructive sleep apnea and the critical role of orofacial growth: evidences. Front Neurol 2013; 3: 184.

3 Guilleminault C, Abad VC, Chiu HY, et al. Missing teeth and pediatric obstructive sleep apnea. Sleep Breath 2016; 20: 561-568.

4 Lee SY, Guilleminault C, Chiu HY, et al. Mouth breathing, nasal "disuse", and pediatric sleep-disordered breathing. Sleep Breath 2015; 19: 1257-1264.

5 Messner AH, Lalakea ML, Aby J, et al. Ankyloglossia: incidence and associated feeding difficulties. Arch Otolaryngol Head Neck Surg 2000; 126: 36-39.

6 Ricke LA, Baker NJ, Madlon-Kay DJ, et al. Newborn tongue tie: prevalence and effect on breast-feeding. $J$ Am Board Fam Pract 2005; 18: 1-7.

7 Hogan M, Westcott C, Griffiths M. Randomized, controlled trial of division of tongue-tie in infants with feeding problems. J Paediatr Child Health 2005; 41: 246-250.

8 García Pola MJ, González García M, García Martín JM, et al. A study of pathology associated with short lingual frenum. ASDC J Dent Child 2002; 69: 59-62.

9 Lalakea L, Messner AH. Ankyloglossia: the adolescent and adult perspective. Otolaryngol Head Neck Surg 2003; 128: 746-752.

10 Marchesan IQ. Lingual frenulum: classification and speech interference. Int J Orofacial Myology 2004; 30: 31-38.

11 Defabianis P. Ankyloglossia and its influence on maxillary and mandibular development. (A seven year follow-up case report). Funct Orthod 2000; 17: 25-33.

12 Martinelli RLC, Marchesan IQ, Berretin-Felix G. Longitudinal study of the anatomical characteristics of the lingual frenulum and comparison to literature. Rev CEFAC 2014; 16: 1202-1207.

13 Martinelli RLC, Marchesan IQ, Berretin-Felix G. Protocol for infants: relationship between anatomic and functional aspect. Rev CEFAC 2013; 15: 599-609.

14 Huang YS, Quo S, Berkowski AJ, et al. Short lingual frenulum and obstructive sleep apnea in children Int $J$ Pediatr Res 2015; 1: 003.

15 Martinelli RLC, Marchesan IQ, Gusmão RJ, et al. Histological characteristics of altered human lingual frenulum. Int J Pediatr Child Health 2014; 2: 5-9.

16 Kotlow LA. Ankyloglossia (tongue-tie): a diagnostic and treatment quandary. Quintessence Int 1999; 30: 259-262.

17 Kotlow LA. Ora diagnosis of abnormal frenum attachments in neonates and infants: evaluation and treatment of the maxillary and lingual frenum using the Erbium: YAG laser. J Pediatr Dental Care 2004; 10: 11-13.

18 Ruffoli R, Giambelluca MA, Scavuzzo MC, et al. Ankyloglossia: a morphofunctional investigation in children. Oral Dis 2005; 11: 170-174.

19 Chervin RD, Hedger K, Dillon JE, et al. Pediatric sleep questionnaire (PSQ): validity and reliability of scales for sleep-disordered breathing, snoring, sleepiness, and behavioral problems. Sleep Med 2000; 1: 21-32.

20 Drake C, Nickel C, Burduvali E, et al. The pediatric sleepiness scale (PDSS): sleep habits and sleep outcome in middle-school children. Sleep 2003; 26: 455-458.

21 Mallampati SR, Gatt SP, Gugino LD et al. A clinical sign to predict difficult tracheal intubation: a prospective study. Can Anaesth Soc J 1985; 32: 429-434.

22 Friedman M, Tanyeri H, La Rosa M, et al. Clinical predictors of obstructive sleep apnea. Laryngoscope 1999; 109: 1901-1907.

23 Friedman M, Ibrahim H, Bass L. Clinical staging for sleep-disordered breathing. Otolaryngol Head Neck Surg 2002; 127: 13-21.

$24 \mathrm{Kim} \mathrm{JH,} \mathrm{Guilleminault} \mathrm{C.} \mathrm{The} \mathrm{nasomaxillary} \mathrm{complex,} \mathrm{the} \mathrm{mandible,} \mathrm{and} \mathrm{sleep-disordered-breathing.} \mathrm{Sleep} \mathrm{Breath}$ 2011; 15: 185-193.

25 Marchesan IQ. Lingual frenulum protocol. Int J Orofacial Myology 2012; 38: 89-103. 
Iber C, Ancoli-Israel S, Chesson AL Jr, et al. The AASM Manual for the Scoring of Sleep and Associated Events: Rules, Terminology and Technical Specifications. Version 2.0. Darien IL, American Academy of Sleep Medicine, 2012 .

27 Arora N, Meskill G, Guilleminault C. The role of flow limitation as an important diagnostic tool and clinical finding in mild sleep-disordered-breathing. Sleep Sci 2015; 8: 134-142.

28 Lin CH, Guilleminault C. Current hypopnea scoring criteria underscore pediatric sleep disordered breathing. Sleep Med 2011; 12: 720-729.

29 Palombini L, Tufik S, Rapoport D, et al. Inspiratory flow limitation in a normal population of adults in São Paulo, Brazil. Sleep 2013; 36: 1663-1668.

30 Lee SY, Guilleminault C, Chiu HY, et al. Mouth breathing, "nasal disuse" and pediatric sleep-disordered breathing Sleep Breath 2015; 19: 1257-1264.

31 Harvold E, Tomer B, Vargervik K, et al. Primate experiments on oral respiration. Am J Orthod 1981; 79: 359-372.

32 Vargervik K, Miller AJ, Chierici G, et al. Morphologic response to changes in neuromuscular patterns experimentally induced by altered modes of respiration. Am J Orthod 1984; 85: 115-124.

33 Marchesan IQ, Martinelli RL, Gusmão RJ. Lingual frenulum: changes after frenectomy. J Soc Bras Fonoaudiol 2012; 24: 409-412.

34 Klockars T. Familial ankyloglossia (tongue-tie). Int J Pediatr Otorhinolaryngol 2007; 71: 1321-1324

35 Packham EA, Brook JD. T-box genes in human disorders. Hum Mol Genet 2003; 12: R37-R44.

36 Braybrook C, Lisgo S, Doudney K, et al. Craniofacial expression of human and murine TBX22 correlates with the cleft palate and ankyloglossia phenotype observed in CPX patients. Hum Mol Genet 2002; 11: 2793-2804

37 Morita H, Mazerbourg S, Bouley DM, et al. Neonatal lethality of LGR5 null mice is associated with ankyloglossia and gastrointestinal distension. Mol Cell Biol 2004; 24: 9736-9743.

38 Kantaputral PN, Paramee M, Kaewkhampa A, et al. cleft lip with cleft palate, ankyloglossia, and hypodontia are associated with TBX22 mutations. J Dent Res 2011; 90: 450-455. 